\title{
High Throughput In Situ EXAFS Instrumentation for the Automatic Characterization of Materials and Catalysts
}

\author{
Nikolaos Tsapatsaris", A. M. Beesley", Norbert Weiher", Moniek Tromp", John

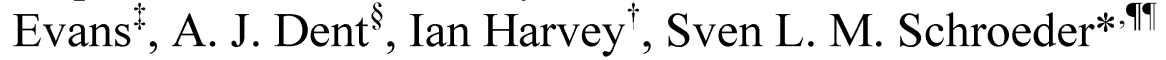 \\ ${ }^{I}$ School of Chemical Engineering and Analytical Science, Molecular Materials Centre, The University of \\ Manchester, Sackville Street, P.O. Box 88. Manchester, M60 1QD, UK. \\ "School of Chemistry, The University of Southhampton, Highfield, Southampton, SO17 1BJ, UK. \\ ${ }^{\S}$ Diamond Light Source Ltd., Diamond House, Chilton, Didcot, Oxfordshire, OX11 ODE, UK. \\ $\uparrow$ Synchrotron Radiation Source (SRS), Daresbury Laboratory, Warrington, Cheshire, WA4 4AD, UK
}

\begin{abstract}
An XAS data acquisition and control system for the in situ analysis of dynamic materials libraries under control of temperature and gaseous environment has been developed. It was integrated at the SRS in Daresbury, UK, beamline 9.3, using a Si (220) monochromator and a 13 element solid state Ge fluorescence detector. The core of the system is an intelligent $\mathrm{X}, \mathrm{Y}, \mathrm{Z}, \theta$ positioning system coupled to multi-stream quadrupole mass spectrometry analysis (QMS). The system is modular and can be adapted to other synchrotron radiation beamlines. The entire software control was implemented using Labview and allows the scan of a variety of library sizes, in several positions, angles, gas compositions and temperatures with minimal operator intervention. The system was used for the automated characterization of a library of 91 catalyst precursors containing ternary combinations of $\mathrm{Cu}$, $\mathrm{Pt}$, and $\mathrm{Au}$ on $\gamma-\mathrm{Al}_{2} \mathrm{O}_{3}$, and for the evaluation and structural characterization of eight $\mathrm{Au}$ catalysts supported on $\mathrm{Al}_{2} \mathrm{O}_{3}$ and $\mathrm{TiO}_{2}$. Mass spectrometer traces reveal conversion rate oscillations in $6 \mathrm{wt} \% \mathrm{Au} / \mathrm{\gamma Al}_{2} \mathrm{O}_{3}$ catalysts. The use of $\mathrm{HT}$ experimentation for in situ EXAFS studies demonstrates the feasibility and potential of HT in situ XAFS for synchrotron radiation studies.
\end{abstract}

Keywords: High throughput, Combinatorial chemistry, XAS, Catalysis, $\mathrm{Au}, \gamma-\mathrm{Al}_{2} \mathrm{O}_{3}$, SBS standard. PACS: 07.85.Qe, 82.65.-s, 61.10.Ht, 81.16.Hc

\section{INTRODUCTION}

The development of intelligent and modular instrumentation is imperative for the identification of catalysts and materials with new target functionalities. Integration of many analytical techniques under a single high throughput (HT) experiment enables detailed screening of numerous candidates and reduces the time scale of the experiments [1]. Parallel experimentation increases the probability of significant discoveries by revealing trends in complex data sets. It increases efficiency and allows for significant reduction of research costs. HT methods have recently become more accepted in the catalytic community, with combinatorial experimentation a promising research avenue for the discovery of new catalysts and optimization of their yield and selectivity [2-4]. The analysis of catalytically active materials requires the use of various scientific methods and experimental setups. Discovering a promising catalyst requires synthesis and in situ screening of sufficiently large, statistically significant libraries. Each catalyst candidate can be defined by a multitude of descriptors (e.g., its constituents, structure, synthetic parameters). which relate to its activity and selectivity and the reaction mechanism.

Various catalyst screening methods have been reported in the literature. Besides kinetic reaction studies, methods such as Infrared spectroscopy [5], Raman spectroscopy [6], X-ray Fluorescence [7], Fluorescence Microscopy [8], Imaging Polarimetry [9], Nuclear Magnetic Resonance spectroscopy [10] and XRD [11] have been incorporated as part of high-throughput setups. These techniques are valuable for characterizing large sample libraries but have limitations, e.g. difficult in situ realization (NMR) or the restriction to crystalline materials (XRD). In catalysis and materials research, EXAFS is used routinely for characterization of the electronic and local

CP879, Synchrotron Radiation Instrumentation: Ninth International Conference, edited by Jae-Young Choi and Seungyu Rah

(C) 2007 American Institute of Physics 978-0-7354-0373-4/07/\$23.00 
geometric structure and for revealing changes in the chemical environment during catalytic reactions [12]. In contrast to the expanding use of HT X-ray diffraction (XRD) [13], the flexibility and inherent advantages of X-ray absorption spectroscopy (XAS) have not been utilized in HT research and present new and promising research ground [14]. XAS characterisation is usually divided into an interpretation of two prominent X-ray absorption finestructure (XAFS) regions in the spectra. The X-ray absorption near-edge structure (XANES) probes the local electronic structure at the site of the X-ray absorbing atoms, whereas the extended X-ray absorption fine-structure (EXAFS) provides structural information, including bond-lengths, coordination numbers as well as static and thermal disorder.

\section{MATERIALS AND METHODS}

\section{Sample Preparation}

The catalysts investigated in this system contained different concentrations of $\mathrm{Au}$ on $\mathrm{Al}_{2} \mathrm{O}_{3}$ and $\mathrm{TiO}_{2}$ supports. $\mathrm{Au} / \mathrm{Al}_{2} \mathrm{O}_{3}$ was prepared using a modified incipient wetness method, $100 \mathrm{mg}$ of $\mathrm{HAuCl}_{4}$ were dissolved in $5.1 \mathrm{ml}$ distilled water, $10 \mathrm{~g}$ of sieved and dried $\mathrm{Al}_{2} \mathrm{O}_{3}$ (Condea, 0.125-0.250 $\mu \mathrm{m}$ ) were added and the system was vigorously shaken for one hour. The resulting gray powder was poured into $200 \mathrm{ml}$ of distilled water and heated to $340 \mathrm{~K}$. The $\mathrm{pH}$ was kept at 8 using $1 \mathrm{M} \mathrm{NaOH}$. After two hours of stirring, the solid was filtered, washed with hot water and dried in vacuum at room temperature for 48 hours. Elemental analysis reported a chlorine content below the detection limit $(<0.2 \mathrm{wt} \%)$. Au/ $/ \mathrm{TiO}_{2}$ was prepared by deposition-precipitation. $400 \mathrm{mg} \mathrm{HAuCl} 4$ were dissolved in $200 \mathrm{ml}$ distilled water. The $\mathrm{pH}$ was adjusted to 7 using $1 \mathrm{M} \mathrm{NaOH} .10 \mathrm{~g}$ of $\mathrm{TiO}_{2}(\mathrm{P} 25$, Degussa) were added and the suspension was heated to $340 \mathrm{~K}$. After two hours of stirring, the solid was filtered and washed with hot water. The resulting powder was dried in vacuum at room temperature for 48 hours. Elemental analysis reported a chlorine content below the detection limit $(<0.2 \mathrm{wt} \%)$.

\section{HT EXAFS Screening}

Our HT XAS data acquisition and control system comprises of an XYZ $\theta$-positioning stage that allows precise positioning of highly compact arrays $\left(>1000\right.$ discrete materials $/ \mathrm{cm}^{2}$ ). Individual gas flows are supplied via independent mass flow controllers (MFC); the effluent of each cell is monitored by quadrupole mass spectrometry (MS). XAS experiments were carried out at station 9.3 at the SRS in Daresbury, UK [15]. The XANES spectra were collected in fluorescence mode using a 13 element Ge solid state detector. Acquisition times were 15 minutes per XANES spectrum. The energy axis was calibrated with a transmission $\mathrm{Au} \mathrm{L}_{3}$-edge spectrum of a Au reference foil. The system was initially tested (without mass spectrometric measurements) using a library of 91 candidates consisting of ternary $\mathrm{Al}_{2} \mathrm{O}_{3}$ supported powder catalyst precursors containing $\mathrm{Cu}, \mathrm{Pt}$, and $\mathrm{Au}$ [16]. For the present experiments a custom made 8 -fold microreactor was used that permitted additional investigations while monitoring the individual conversions using a QMS through 1/16" connections (Omnifit) Au $\mathrm{L}_{\text {III }}$ XANES spectra were obtained from the catalysts during temperature programmed reduction (TPR) under CO (Purity 3.8, BOC) atmosphere. Subsequently the samples were exposed to various $\mathrm{CO}: \mathrm{O}_{2}(99.99 \%)$ : $\mathrm{He}(99.99 \%)$ mixtures and spectra were acquired in situ. Data reduction was performed using the IFEFFIT library and the corresponding front ends ATHENA \& ARTEMIS [17].

\section{RESULTS \& DISCUSSION}

Figure 1(left) demonstrates interrelations between the different components of the HT setup and the beamline. Positioning control was accomplished using a $\pm 10 \mu \mathrm{m}$ step 4 degree of freedom mechanical apparatus composed of three linear stages (Parker) and a 1 circle segment (Huber). The stages were stacked in three vertical planes providing range of movement of $400 \times 200 \times 300 \mathrm{~mm}(\mathrm{x}, \mathrm{y}, \mathrm{z})$ and $\pm 10^{\circ}$ rotation around the $\mathrm{y}$ axis. The mechanical setup was mounted on the existing optical table of the SRS station and required virtually no setup changes. The sample library was positioned in such way so that the procedure of loading samples is more efficient by instructing the software to position the in situ cell closer to the operator. Additional adjustments were made to the design to accommodate for space restrictions in the beamline, for example the small distance of the hutch wall to the X-ray beam $(125 \mathrm{~mm})$. The gas and MFC manifold (Bronkhorst) permitted parallel control of four gases $\left(\mathrm{CO}, \mathrm{O}_{2}, \mathrm{He}, \mathrm{H}_{2}\right)$ 
to within $1 \%$ at the maximum flow $100 \mathrm{ml} / \mathrm{min} /$ gas through the in situ cell. Stainless steel tubing (1/4") was used for interconnecting the MFCs and gas cylinders. Various protocols, including the industry-standard Profibus were incorporated for flexibility and expandability (Fig. 2 right). Combining the RS232 and CAN protocols and a custom made driver allowed communication with the positioning stages and the rest of the components of the HT setup. Data acquisition cards were used to provide the necessary analogue and digital lines to the MFCs and solenoid valves. Synchronization with the station control computer was provided via a TCP/IP network connection with a custom made handshake protocol. The protocol contains commands for the start, stop and mode of EXAFS scans, adjusting monochromator position, scan downloading and also more detailed calibration routines. The main control computer hosts an in-house LABVIEW program that is responsible for automatic positioning of the 8 well array in any position or angle with respect to the X-ray beam, allowing accurate measurements of XAFS data for each individual catalyst or material. The program is built around libraries responsible for the individual control of every component such as multiple temperature control, valve manifolds, mass flow controllers, yet maintaining compatibility with the various instrumentation infrastructures present at different synchrotron radiation beamlines.
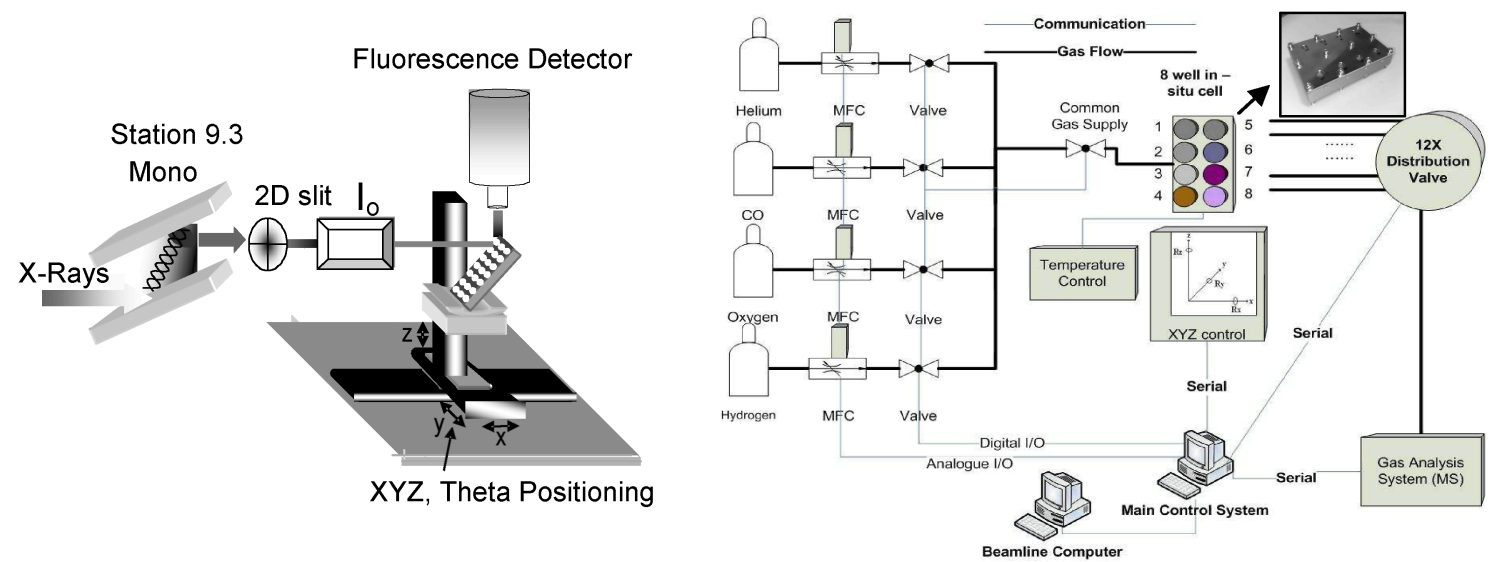

FIGURE 1. Integration of HT in situ EXAFS instrumentation in Station 9.3 of SRS Daresbury. Left: Robotic stage with respect to the station detector and X-ray beam, Right: Main system components and communication interface with beamline. The inset shows the custom made 8 well reactor.

Initial examination of the XANES spectra of Au catalysts revealed saturation effects at high metal concentrations. A linear correlation between edge step and nominal Au concentration was found up to a metal loading of approximately $4 \mathrm{wt} \%$. The spectral distortions at higher Au concentrations can be attributed to selfabsorption effects and/or a non linear response of the detector. The development of the chemical state of the Au component during temperature programmed reduction (TPR) of one of the catalysts in the 8 well reactor can be seen on Fig 2 (left). It is seen how $\mathrm{Au}^{3+}$ was reduced in an (1/1) $\mathrm{CO} / \mathrm{He}$ stream, as indicated by a decrease of the nearedge feature at $11925 \mathrm{eV} \mathrm{[18].} \mathrm{It} \mathrm{shall} \mathrm{be} \mathrm{noted} \mathrm{that} \mathrm{the} \mathrm{material} \mathrm{was} \mathrm{already} \mathrm{partially} \mathrm{reduced} \mathrm{before} \mathrm{exposure} \mathrm{to}$ $\mathrm{CO}$ as indicated by the low whiteline intensity. Fig 2 (right) shows $\mathrm{MS}$ transients of $\mathrm{O}_{2}, \mathrm{CO}, \mathrm{He}$ and $\mathrm{CO}_{2}$ during $\mathrm{CO}$
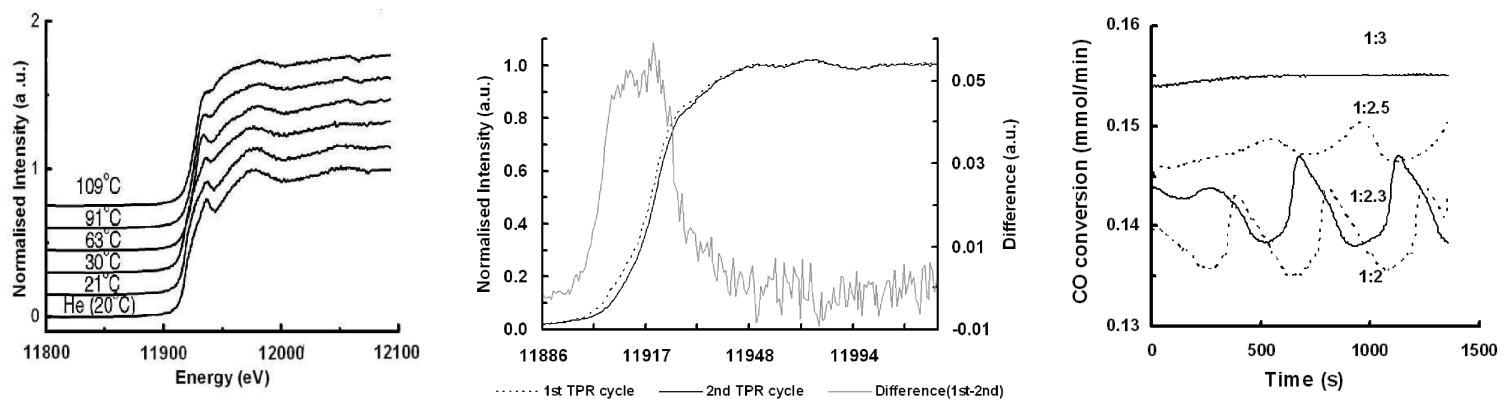

FIGURE 2. Left: $\mathrm{Au} \mathrm{L}_{\mathrm{III}} \mathrm{XANES}$ series of Temperature Programmed Reduction of $4 \% \mathrm{Au} / \mathrm{Al}_{2} \mathrm{O}_{3}$ on a CO:He (1:1) stream. Centre: XANES Spectra of $4 \% \mathrm{Au} / \mathrm{Al}_{2} \mathrm{O}_{3}$ during $\mathrm{CO}$ oxidation with 1:2 $\mathrm{CO}: \mathrm{O} 2$ after two reduction cycles. Oscillations on this sample were observed only after the first reduction cycle. Right: Non linear CO conversion response of $6 \% \mathrm{Au} / \mathrm{Al}_{2} \mathrm{O}_{3}$. Sustained oscillations were observed at $\mathrm{CO} / \mathrm{O}_{2}$ ratios between 0.4 and 0.625 . 
oxidation over a $1 \% \mathrm{Au} / \mathrm{Al}_{2} \mathrm{O}_{3}$ catalyst. An oscillatory response of $\mathrm{CO}$ conversion during $\mathrm{CO}$ oxidation was first identified on a $4 \% \mathrm{Au} / \mathrm{Al}_{2} \mathrm{O}_{3}$ catalyst (Fig. 2, center) under a $\mathrm{CO}: \mathrm{O}$ 1:2 gas mixture and after a deactivation period of 6 hours. Reproduction of the short-lived oscillatory response was not successful after a $2^{\text {nd }}$ TPR cycle. The difference XANES spectrum shown in Fig. 2 (center) indicates the presence of a $-1 \mathrm{eV}$ edge shift between the oscillating and steady state catalysts. The appearance of a shoulder at $-3 \mathrm{eV}$ could be attributed to weak oxidation by adsorbed gas species and is in agreement with previous observations [19]. Conversion oscillations similar to those observed here were previously observed in single crystal transition metal catalysts, e.g. for Pt, Rh and Ir [20-24]. Usually, oscillations are related to reduction/oxidation cycles, resulting, for example, in non linear rate phenomena arising from oxide or sub-surface layers that deactivate slowly the reactive surface [24]. Preliminary XANES analysis indicates that such oxidation/reduction cycles maybe present on the Au component of our catalysts (Fig. 2, center). The $15 \mathrm{~min}$ delay in the acquisition of XANES spectra caused by the acquisition electronics of the fluorescent detector as well as during the experiments did not allow for resolving the chemical state of the catalyst within an oscillation cycle.

In summary, integration of the HT system in the synchrotron station facilitated the rapid acquisition of ex situ and in situ XAS data with parallel MS, gas-flow and temperature control. This parallel control system has already facilitated the discovery of $\mathrm{CO}$ oxidation rate oscillations over Au catalysts. The results demonstrate the advantages of HT experimentation when used in situ EXAFS studies.

\section{ACKNOWLEDGMENTS}

This work was supported through EPSRC research grants GR/S85801/01 and GR/S85818/01. The CCLRC Synchrotron Radiation Source (SRS) at Daresbury Laboratory is acknowledged for providing beamtime at station 9.3 under award number 44135 .

\section{REFERENCES}

1. R. Hoogenboom, F. Wiesbrock, M A.M. Leenen, M A.R. Meier, U S. Schubert, J. Comb. Chem 7, 10-13 (2005)

2. R.A. Potyrailo, W.J. Morris,R.J. Wroczynski, P.J. McCloskey, J. Comb. Chem 9, 869-873 (2004)

3. A. Potyrailo, R. J. Wroczynski, J. P. Lemmon, W. P. Flanagan, O. P. Siclovan, J. Comb. Chem 5, 8-17 (2003)

4. R. Malhotra, Ed. Combinatorial Approaches to Materials Development; ACS Symposium Series 814; American Chemical Society: Washington DC, 2002.

5. A. Venimadhav, K.A. Yates, M. G. Blamire, J. Comb. Chem 7, 85-89 (2005)

6. A. Leugers, David R. Neithamer, Larry S. Sun, Jack E. Hetzner, Sean. Hilty, Sam Hong, Matthew Krause,Kenneth. Beyerlein, J. Comb. Chem 5, 238-244 (2003)

7. T. C. Miller, Grace Mann, George J. Havrilla, Cyndi A. Wells, B. P Warner, Tom R, Baker, J. Comb. Chem 5, 245-252 (2003)

8. M. M. Taniguchi, R.A. Farrer, J. Fourkas, J. Comb. Chem 7, 54-57 (2005)

9. P. R.Gibbs, C. S. Uehara, P. T. Nguyen, R.C. Willson, Biotechnol. Prog.19, 1329-1334 (2003)

10. R.A. Kautz, W.K Goetzinger, B.L. Karger, J. Comb. Chem 7, 14-20 (2005)

11. J.H. Reibenspies, N.S.P. Bhuvanesh, Journal of Pharmaceutical and Biomedical Analysis 37, 611-614 (2005)

12. J. J. Rehr, A. L. Ankudinov, S. I. Zabinsky, Catal. Today 39, 263 (1998).

13. A. E. Russell, A. Rose, Chemical Reviews 104, 4613 (2004)

14. A. Corma, J. M. Serra, P. Serna, M. Moliner, Journal of Catalysis 232, 335 (2005)

15. A. J. Dent, G. Derst, G. E. Van Dorssen, J. F. M. Mosselmans, Daresbury Synchrotron Radiation Source (SRS), Manual Station 9.3. http://www.srs.dl.ac.uk/srs/stations/station9.3.htm.

16. N. Tsapatsaris, A. Beesley, N. Weiher, M. Tromp, J. Evans, A. Dent, I. Harvey, S. Hayama, S. L.M. Schroeder to be submitted to J. Comb. Chem.

17. M. J Newville, J. Synchrotron Rad 8, 322 (2001)

18. N. Weiher, E. Bus, L Delannoy, C. Louis, D. E. Ramaker, J. T. Miller, J. A. van Bokhoven, Journal of Catalysis 240, (2), 100-107 (2006)

19. J. A. van Bokhoven, C. Louis, J. T. Miller, M. Tromp, O. V. Safonova, P. Glatzel, Angew. Chem. Int. Ed., DOI: 10.1002/anie.200123456 in press

20. P. A. Carlsson et al, Journal of Catalysis 226, (2), 422-434 (2004)

21. R. Danielak, A. Perera, M. Moreau, M. Frankowicz, R. Kapral Physica A 229, (3-4), 428-443 (1996)

22. V. V. Gorodetskii, V. I. Elokhin, J. W. Bakker, B. E. Nieuwenhuys, Catalysis Today 105, (2), 183-205 (2005)

23. L. Li, W. Wlodarski, K. Galatsis, D. A. Powell, Sensors and Actuators B: Chemical 96, (3), 610-614 (2003)

24. C. D. Lund, C. M. Surko, M. B. Maple, S. Y. Yamamoto, Surface Science 459, (3), 413-425 (2000) 